\title{
Densitometry of Active Star Forming Galaxies
}

\author{
C. Henkel ${ }^{1}$, J. G. Mangum ${ }^{2}$, J. Darling ${ }^{3}$ and K. M. Menten ${ }^{4}$ \\ ${ }^{1}$ Max-Planck-Institut für Radioastronomie, Auf dem Hügel 69, 53121 Bonn, Germany \\ Astron. Dept., King Abdulaziz University, P.O. Box 80203, Jeddah, Saudi Arabia \\ email: chenkel@mpifr-bonn.mpg.de \\ ${ }^{2}$ National Radio Astronomy Observatory, 520 Edgemont Road, \\ Charlottesville, VA 22903, USA \\ email: jmangum@nrao.edu \\ ${ }^{3}$ Center for Astrophysics and Space Astronomy, Department of Astrophysical and Planetary \\ Sciences, Box 389, University of Colorado, Boulder, CO 80309-0389, USA \\ email: jdarling@origins. colorado.edu \\ ${ }^{4}$ Max-Planck-Institut für Radioastronomie, Auf dem Hügel 69, 53121 Bonn, Germany \\ email: kmenten@mpifr-bonn.mpg.de
}

\begin{abstract}
Attempting to distangle density and kinetic temperature of the star forming molecular gas in the central regions of galaxies, we have embarked on a project involving sensitive measurements of a variety of formaldehyde $\left(\mathrm{H}_{2} \mathrm{CO}\right)$ and ammonia $\left(\mathrm{NH}_{3}\right)$ transitions. Preliminary results, based on observations from the Green Bank Telescope (GBT) and the Very Large Array (VLA) are summarized and an outline for the entire project is given.
\end{abstract}

Keywords. ISM: molecules — galaxies: nuclei — galaxies: starburst — radio lines: galaxies

\section{Introduction}

To study the physical properties of the dense star forming molecular gas, abundant, high-dipole moment molecules are the best tracers. For this reason, emission line studies from linear molecules like $\mathrm{CS}, \mathrm{HCN}, \mathrm{HCO}^{+}$or $\mathrm{HNC}$ have been carried out toward a variety of nearby galaxies (e.g., Henkel et al. 1991; Gao \& Solomon 2004a,b). However, simultaneous determinations of density and kinetic temperature suffer from a degeneracy. Observed line intensities can be fitted by both low densities and high kinetic temperatures and by high densities and low kinetic temperatures. To obtain a better understanding of the physical parameters of the dense star forming gas in the inner parts of active galaxies, we have therefore started a program based on spectra from the non-linear molecules $\mathrm{H}_{2} \mathrm{CO}$ and $\mathrm{NH}_{3}$, making use of recent drastic improvements with respect to attainable bandwidths and sensitivities.

\section{The molecules}

$\mathrm{H}_{2} \mathrm{CO}$ : Formaldehyde $\left(\mathrm{H}_{2} \mathrm{CO}\right)$ has proven to be a reliable density and kinetic temperature probe for galactic molecular clouds. Being a slightly asymmetric rotor, most rotational levels are split into two states. This splitting leads to two types of transitions, the high frequency (often mm-wave) $\Delta J=1$ rotational and the lower frequency (often cm-wave) $\Delta J=0 \mathrm{~K}$-doublet lines, which connect the two states of a split rotational level ( $J$ : total angular momentum quantum number, $K$ : projection of $J$ on the molecular axis). The K-doublet lines have a unique property. For $n\left(\mathrm{H}_{2}\right) \lesssim 10^{5} \mathrm{~cm}^{-3}$, the lower energy 
states of the $J_{\mathrm{K}_{\mathrm{a}} \mathrm{K}_{\mathrm{c}}}=1_{10} \rightarrow 1_{11}$ to $5_{14} \rightarrow 5_{15} \mathrm{~K}$-doublet transitions become overpopulated due to a collisional selection effect (Evans et al. 1975) and are therefore seen in absorption even against the microwave background. At higher densities, the lines get thermalized and are seen in emission. Measurements of these K-doublet lines as well as of rotational lines within a $K_{\mathrm{a}}$-ladder are good measures of density (if $T_{\text {kin }}$ is known), with the collisional selection effect providing an important additional constraint. A comparison of ortho- or para- $\mathrm{H}_{2} \mathrm{CO}$ lines from different $K_{\mathrm{a}}$-ladders leads instead to a determination of kinetic temperature, which does not require any a priori knowledge of density.

$\mathrm{NH}_{3}: \mathrm{NH}_{3}$, a symmetric rotor, shows (like $\mathrm{H}_{2} \mathrm{CO}$ ) split rotational levels in the $K>0$ ladders. In this case it is due to the quantum mechanical tunneling of the nitrogen atom through the plane of the three hydrogen atoms. Most transitions connecting the two states of such inversion doublets are near $\lambda \sim 1 \mathrm{~cm}$ and cover a wide range of excitation. Metastable $(J=K)$ levels of ortho- or para- $\mathrm{NH}_{3}$ are connected via collisions. Thus their relative intensities provide good estimates of kinetic temperature, without requiring an a priori knowledge of the density. Complementing such data, non-metastable $(J>K)$ transitions can be used to trace the infrared radiation field in star forming regions. For $n\left(\mathrm{H}_{2}\right) \lesssim 10^{7} \mathrm{~cm}^{-3}$, excitation temperatures remain low. However, in the presence of a strong infrared radiation field, level populations of the $(J, K)=(2,1)$ line (i.e., the line connecting the upper with the lower state in the $(2,1)$ inversion doublet) become inverted, providing a sensitive probe of the radiation environment (Morris et al. 1973).

\section{Source selection and $\mathrm{H}_{2} \mathrm{CO}$ measurements with the GBT}

Our survey, initially using the Green Bank Telescope (GBT), includes 56 galaxies with $\delta>-40^{\circ}, L_{\mathrm{IR}}>10^{9} \mathrm{~L}_{\odot}, L_{60 \mu \mathrm{m}}>50 \mathrm{Jy}$, and/or bright $\mathrm{HCN}$ and $\mathrm{CO}$ emission. The galaxies include nearby sources like NGC 253, IC 342, and M 82 at $D \sim 3-4 \mathrm{Mpc}$, but also much more distant (ultra)luminous infrared galaxies ((U)LIRGs) like Arp 220 or UGC 5101 at $D \sim 70$ and $100 \mathrm{Mpc}$.

These sources were observed with the GBT in the $6 \mathrm{~cm} 1_{10} \rightarrow 1_{11}$ and the $2 \mathrm{~cm} 2_{11} \rightarrow 2_{12}$ K-doublet transitions. Early results were published by Mangum et al. (2008). Up to now, both transitions have been detected in a total of 13 galaxies, almost tripling the number of previously known such sources. Furthermore, 26 galaxies were detected in at least one of the two transitions. As indicated in Sect. 2, emission in either transition of this study indicates high spatial densities $\left(n\left(\mathrm{H}_{2}\right)>10^{5} \mathrm{~cm}^{-3}\right)$ as, e.g., seen toward IRAS 15107+0724 (Fig. 1, left panel). Absorption in both $\mathrm{H}_{2} \mathrm{CO}$ transitions as seen in NGC 253 hints at lower densities.

Analyzing these data with a Large Velocity Gradient (LVG) model and assuming $T_{\text {kin }}=T_{\text {dust }} \sim 30-40 \mathrm{~K}$ (values for (U)LIRGs tend to be slightly higher), as the kinetic temperature of the gas, it turns out that apparently higher mean densities are found in the (U)LIRGs, while lower mean densities prevail in the less luminous targets.

\section{Ammonia $\left(\mathrm{NH}_{3}\right)$ measurements with the GBT}

Our assumption of $T_{\text {dust }}=T_{\text {gas }}$ may not be correct. In environments energetically dominated by photons, X-rays, cosmic rays or turbulent heating, gas temperatures may significantly surpass dust temperatures, at least if densities are not exorbitantly high. Therefore, $T_{\text {kin }}$ values from the gas phase are an essential parameter, not only to estimate densities from $\mathrm{H}_{2} \mathrm{CO}$, but also to evaluate previously taken data from the abundant linear molecules mentioned in Sect. 1. 

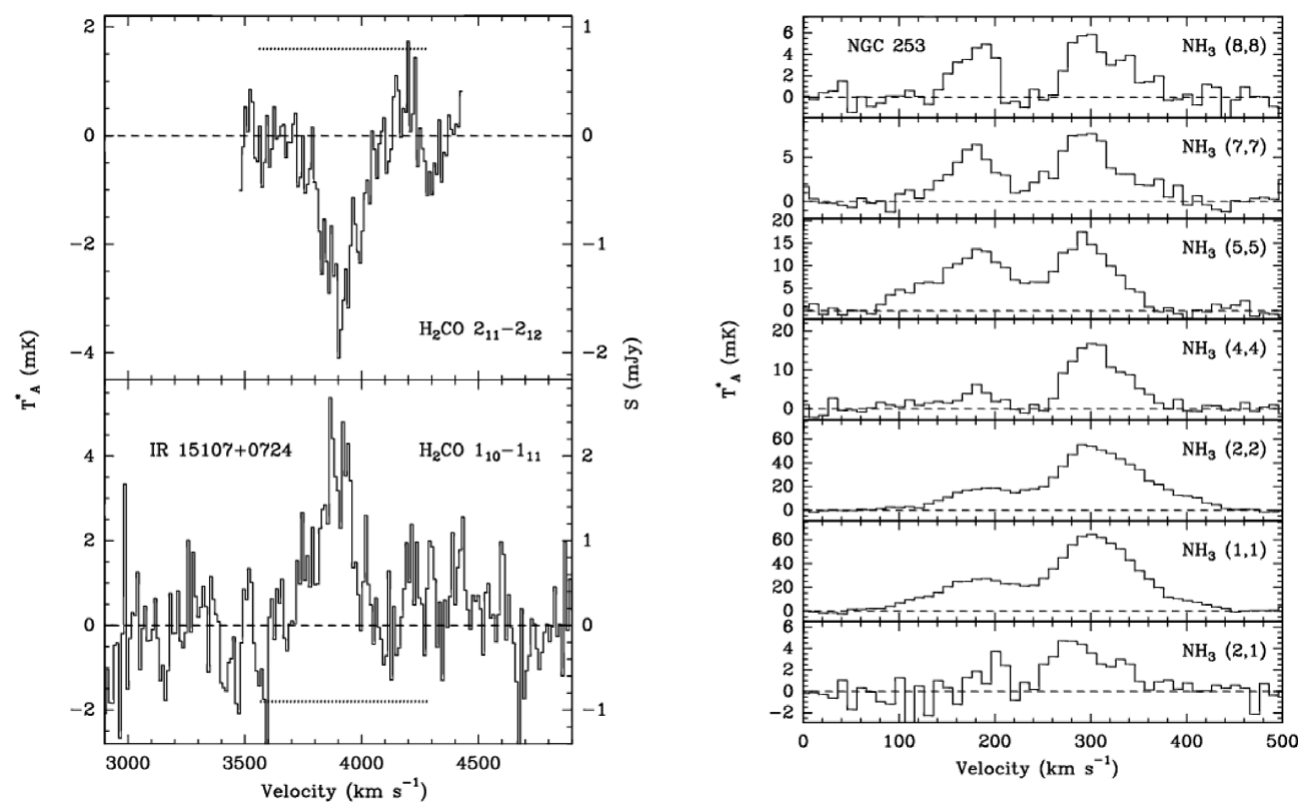

Figure 1. Left panel: $6 \mathrm{~cm}$ (lower spectrum) and $2 \mathrm{~cm}$ (upper spectrum) of $\mathrm{H}_{2} \mathrm{CO}$ toward the ULIRG IRAS $15107+0724$. Right panel: $\mathrm{NH}_{3}$ lines observed toward the nearby starburst galaxy NGC 253. Note the detection of the non-metastable $(2,1)$ inversion line.

With the GBT we can observe up to four $\mathrm{NH}_{3}$ inversion lines simultaneously. Two optimized observing configurations allow for measurements of the metastable $(1,1)$ to $(7,7)$ and the non-metastable $(2,1)$ transitions (the $(8,8)$ and $(9,9)$ lines were also measured in exceptional cases). The $(7,7)$ line is $\sim 535 \mathrm{~K}$ above the ground state. 19 of the 26 galaxies detected in $\mathrm{H}_{2} \mathrm{CO}$ have already been targeted, detecting at least the $(1,1)$ and $(2,2)$ lines in 12 objects (e.g., Fig. 1, right panel). For the first time, extragalactic ammonia has been detected in a non-metastable inversion doublet. The $(2,1)$ line is seen toward five sources. Derived $T_{\text {kin }}$ values surpass $T_{\text {dust }}$ values in many sources, in particular in those with high infrared luminosities. As a consequence, the correlation between density and $L_{\mathrm{IR}}$, suggested in Sect. 2 by assuming $T_{\text {dust }}=T_{\text {kin }}$, does not hold any more. Densities of the bulk of the molecular gas cover the rather small range $10^{4.5} \mathrm{~cm}^{-3}<n\left(\mathrm{H}_{2}\right)<$ $10^{5.5} \mathrm{~cm}^{-3}$. This implies that the Schmidt-Kennicutt relation between $L_{\mathrm{IR}}$ and $M_{\mathrm{dense}}$, the mass of the dense gas, is (1) an indication of the dense gas mass reservoir available to form stars, and (2) is not directly dependent upon a higher average density driving the star formation process in the most luminous starburst galaxies.

\section{Occam's Razor and High Resolution Measurements}

Using the simplest possible interpretation, we have so far relied on beam averaged quantities. While such an approach is reasonable for first order estimates, it ignores the potential impact of masers, distorting the picture outlined above. This holds in particular for $\mathrm{H}_{2} \mathrm{CO}$, where Baan et al. (1986) proposed $6 \mathrm{~cm}$ maser emission in the case of Arp 220, but also for $\mathrm{NH}_{3}$, where Ott et al. (2005) and Lebrón et al. (2011) discuss tentatively detected extragalactic $(3,3)$ and $(6,6)$ masers.

With our LVG codes, all data could be successfully simulated without having to adopt population inversion. However, GBT data alone do not provide a tool to discriminate safely between quasi-thermal and maser emission. With the VLA, it will be possible, (1) 
to reject or to confirm the presence of masers in individual sources and (2) to reveal spatial fine structure. First data are presently being collected.

\section{Outline}

While the project is still far from completion, the steps necessary to derive the densities and kinetic temperatures in the nuclear regions of active galaxies are quite clear. There are seven major steps:

- Measurements of the 6 and $2 \mathrm{~cm} \mathrm{H}_{2} \mathrm{CO}$ lines with the GBT

- Observations of eight $\mathrm{NH}_{3}$ transitions with the GBT

- Follow-up measurements of the galaxies detected in $\mathrm{H}_{2} \mathrm{CO}$ with the VLA

- Follow-up measurements of the galaxies detected in $\mathrm{NH}_{3}$ with the VLA

- Measurements of rotational $\mathrm{H}_{2} \mathrm{CO}$ lines to determine densities with IRAM/ALMA

- Determinations of kinetic temperatures through $\mathrm{H}_{2} \mathrm{CO}$ observations with ALMA

- Apply the gained knowledge on kinetic temperatures also to data from other molecules

While the first four steps have already been explained above, we have also carried out IRAM 30-m measurements of rotational transitions of $\mathrm{H}_{2} \mathrm{CO}$ toward a couple of galaxies. It is worthwhile to note that $\mathrm{H}_{2} \mathrm{CO}$ is particularly useful, because its fractional abundance in molecular clouds does not vary strongly (e.g., Johnstone et al. 2003), so that $\mathrm{H}_{2} \mathrm{CO}$ traces a representative fraction of the entire dense molecular gas phase. In view of this unique advantage, which is not shared by $\mathrm{NH}_{3}$, it would be highly desirable to also determine kinetic temperatures from $\mathrm{H}_{2} \mathrm{CO}$ for a large number of galaxies. This has not been done so far. While Mühle et al. (2007) presented a pioneering study of M 82, observing the $K_{\mathrm{a}}=0$ and 2 triplet near $218 \mathrm{GHz}$, extreme sensitivities are required for almost all other targets. The Atacama Large Millimeter/Submillimeter Array (ALMA) has the required collecting area to perform such measurements. We then would have reliable density and kinetic temperature estimates from a single tracer, which samples rather evenly the molecular gas phase. Using the resulting kinetic temperatures, density estimates could then also be deduced from other molecules like those mentioned earlier (see Sect. 1). Note, however, that $n\left(\mathrm{H}_{2}\right)$ and $T_{\text {kin }}$ derived from just one very representative species, namely $\mathrm{H}_{2} \mathrm{CO}$, would likely continue to provide the most valuable estimates of these important physical parameters.

\section{References}

Baan, W. A., Güsten, R., \& Haschick, A. D. 1986, ApJ, 305, 830

Evans, N. J., Zuckerman, B., Morris, G., \& Sato, T. 1975, ApJ, 196, 433

Gao, Y. \& Solomon, P. M. 2004a, ApJS, 152, 63

Gao, Y. \& Solomon, P. M. 2004b, ApJ, 606, 271

Henkel, C., Baan, W. A., \& Mauersberger, R. 1991, A\&AR, 3, 47

Johnstone, D., Boonman, A. M. S., \& van Dishoeck, E. F. 2003, A\& $A, 412,157$

Lebrón, M., Mangum, J. G., Mauersberger, R., et al. 2011, A $\mathfrak{E} A$, 534, A56

Mangum, J. G., Darling, J., Menten, K. M., \& Henkel, C. 2008, ApJ, 673, 832

Morris, M., Zuckerman, B., Palmer, P., \& Turner, B. E. 1973, ApJ, 186, 501

Mühle, Seaquist, E. R. \& Henkel, C. 2007, ApJ, 671, 1579

Ott, J., Weiß, A., Henkel, C., \& Walter, F. 2005, ApJ, 629, 767 